\title{
COUNTABLE PARACOMPACTNESS IN LINEARLY ORDERED SPACES
}

\author{
B. J. BALL
}

A Hausdorff space $X$ is said to be paracompact [2] provided that if $W$ is a collection of open sets covering $X$, there exists a collection $W^{\prime}$ of open sets covering $X$ such that (1) each element of $W^{\prime}$ is a subset of an element of $W$ and (2) each point of $X$ belongs to an open set which intersects only a finite number of elements of $W^{\prime} ; X$ is said to be countably paracompact [3] provided that if $W$ is a countable collection of open sets covering $X$, there exists a collection $W^{\prime}$ satisfying the above conditions. It is known that not every normal Hausdorff space is paracompact [2], but the question whether every such space is countably paracompact is as yet unsolved (cf. [3]). Since every linearly ordered space ${ }^{1}$ is a normal Hausdorff space (cf. $[1$, p. 39]) but not necessarily paracompact [2], it seems natural to inquire whether every linearly ordered space must be countably paracompact. The purpose of the present note is to show that this is the case.

Definitions. 1. A collection $G$ of subsets of a space $X$ is said to be locally finite provided every point of $X$ belongs to an open set $X$ which intersects at most a finite number of the elements of $G$. 2. If $G$ and $H$ are collections of sets, then $H$ is said to be a refinement of $G$ provided each element of $H$ is a subset of some element of $G$. 3. A collection $G$ of sets is said to be coherent provided $G$ is not the sum of two collections $G_{1}$ and $G_{2}$ such that no element of $G_{1}$ intersects an element of $G_{2}$. 4. If $p$ belongs to some element of the collection $G$ of sets, then the star of $p$ with respect to $G$ is the sum of the elements of $G$ which contain $p$.

Suppose $X$ is a linearly ordered space and $W$ is a countable collection of open sets covering $X$. For each point $p$ of $X$, let $M_{p}$ denote the set of all points $x$ of $X$ such that there is a finite, coherent collection $H_{x}$ of open intervals of $X$ such that $H_{x}$ is a refinement of $W$ and covers the set whose elements are $p$ and $x$. For each point $p$ of $X$, let $K_{p}$ denote the collection of all open intervals $k$ of $X$ such that $k$ is a subset of $M_{p}$ and of some element of $W$. It is easily seen that for each $p$ in $X, M_{p}$ is both open and closed and if $q \in M_{p}$, then $M_{q}=M_{p}$.

Presented to the Society, June 20,1953, under the title $A$ note on paracompactness; received by the editors May 14, 1953 and, in revised form, July 18, 1953.

${ }^{1}$ By a linearly ordered space is mean a simply ordered set with its intrinsic topology: i.e., the topology in which "neighborhood of $x$ " means "open interval containing $x$." 
Hence if for each point $p$ of $X$, there is a locally finite collection $G_{p}$ of open sets which is a refinement of $K_{p}$ and covers $M_{p}$, then there is a locally finite collection $G$ of open sets which is a refinement of $W$ and covers $X$. The existence of such a collection $G_{p}$ for each $p$ in $X$ is a consequence of the following two theorems.

Theorem 1. If $p \in X$ and $R=\left\{x \in M_{p} \mid p \leqq x\right\}$, then either (1) $R$ is covered by a finite subcollection of $K_{p}$ or (2) there is a sequence $\left\{x_{n}\right\}$ of points of $R$ such that, for each $n, x_{n}<x_{n+1}$ and if $x \in R$, then, for some $n, x<x_{n}$.

Proof. Let $G_{0}$ denote the star of $p$ with respect to $K_{p}$. If there is a point of $R$ which is preceded ${ }^{2}$ by every point of $G_{0}$, then there is a point $a_{1}$ of $R$ which is preceded by every point of $G_{0}$ and which belongs to an element of $K_{p}$ which intersects $G_{0}$. Let $G_{1}$ denote the star of $a_{1}$ with respect to $K_{p}$. If there is a point of $R$ which is preceded by every point of $G_{1}$, then there is a point $a_{2}$ of $R$ which is preceded by every point of $G_{1}$ and which belongs to an element of $K_{p}$ which intersects $G_{1}$. It follows by induction that there exist (possibly finite) sequences $G_{0}, G_{1}, G_{2}, \cdots$ and $a_{0}, a_{1}, a_{2}, \cdots\left(a_{0}=p\right)$ such that no point of $R$ is preceded by every point of $\sum G_{i}$ and for each $n$, (1) $a_{n}$ is a point of $R$, (2) $G_{n}$ is the star of $a_{n}$ with respect to $K_{p}$, and (3) $a_{n+1}$ is preceded by every point of $G_{n}$ and belongs to an element of $K_{p}$ which intersects $G_{n}$. Let $\alpha$ denote the sequence $G_{0}, G_{1}, G_{2}, \cdots$.

Suppose $k$ is an element of $K_{p}$ which intersects $R \cdot \sum G_{i}$. Let $j$ denote the smallest integer $n$ such that $k$ intersects $G_{n}$. Suppose $G_{j}$ is the last term of $\alpha$. Then $G_{j}$ contains $\left\{x \in M_{p} \mid a_{j} \leqq x\right\}$. If $j=0$, then, since $k$ intersects $R, k$ either contains $a_{0}$ or is a subset of $R$; in either case $k$ is a subset of $G_{0}$. Suppose $k \neq 0$. If $k$ contains a point $x$ such that $a_{j} \leqq x$, then $k$ is a subset of $G_{j}$. If each point of $k$ precedes $a_{j}$, then, since there is an element of $K_{p}$ which contains $a_{j}$ and intersects $G_{j-1}$ and $k$ does not intersect $G_{j-1}, k$ is a subset of $G_{j}$. Suppose $G_{j}$ is not the last term of $\alpha$. Then if $k$ is not a subset of $G_{j}+G_{j+1}$, it contains a point which is preceded by every point of $G_{j+1}$ and hence, since $k$ intersects $G_{j}, k$ contains $a_{j+1}$. But this implies that $k$ is a subset of $G_{j+1}$. Thus every element of $K_{p}$ which intersects $R \cdot \sum G_{i}$ is a subset of $\sum G_{i}$.

If $\alpha$ is infinite, for each $n$, let $x_{n}=a_{n}$; it is easily seen that $\left\{x_{n}\right\}$ satisfies condition (2) of the conclusion of this theorem. Suppose $\alpha$ is finite. Let $G_{j}$ be the last term of $\alpha$ and let $D=\left\{x \in M_{p} \mid a_{j}<x\right\}$. Since $D$ is an open subset of $M_{p}$, if $D$ is a subset of any element of $W$, condition (1) is satisfied. Suppose $D$ is not a subset of any element

2 If $x<y$, then $x$ is said to precede $y$. 
of $W$. Let $w_{1}, w_{2}, w_{3}, \cdots$ denote the elements of $W$ which contain $a_{j}$. For each $n$, let $T_{n}$ denote the sum of all elements of $K_{p}$ which contain $a_{j}$ and lie in $w_{n}$. For each $n$, there is a point of $M_{p}$ which is preceded by every point of $T_{n}$ (otherwise $D$ would be a subset of $w_{n}$ ) and hence there exists a sequence $\left\{x_{n}\right\}$ of points of $R$ such that $x_{1}$ is preceded by every point of $T_{1}$ and for each $n, x_{n+1}$ is preceded by each of the points $x_{1}, x_{2}, \cdots, x_{n}$ and by every point of $T_{n}$. Suppose $x$ is a point of $R$. If $x$ belongs to $G_{j}$, it belongs to an element $k$ of $K_{p}$ which contains $a_{j}$. Since $k \in K_{p}$, for some $n, k$ is a subset of $w_{n}$ and hence of $T_{n}$. Consequently $x<x_{n+1}$. If $x$ does not belong to $G_{j}$, then $x<a_{j}$ and hence $x<x_{1}$. Hence condition (2) is fulfilled.

ThEOREM 2. Under the hypothesis of Theorem 1, there is a locally finite collection $U$ of open sets which is a refinement of $K_{p}$ and covers $R$.

Proof. If condition (1) of the conclusion of Theorem 1 is satisfied, there is such a collection $U$. Suppose Condition (1) is not satisfied and let $\left\{x_{n}\right\}$ be a sequence of points of $R$ satisfying condition (2). For each $n$, let $H_{n}$ denote a finite, coherent collection of elements of $K_{p}$ which covers the set whose elements are $p$ and $x_{n+1}$. Let $H_{1}^{\prime}=H_{1}$ and for each $n$ greater than 1 , let $H_{n}^{\prime}$ denote a finite collection of open intervals such that (1) $H_{n}^{\prime}$ is a refinement of $H_{n}$ which covers the closed interval $x_{n} x_{n+1}$ and (2) no element of $H_{n}^{\prime}$ intersects the closed interval $p x_{n-1}$. Let $U=\sum H_{i}^{\prime}$. Then $U$ is a locally finite collection of open sets which is a refinement of $K_{p}$ and covers $R$.

It can be shown by a similar argument that if $L=\left\{x \in M_{p} \mid x \leqq p\right\}$, then there is a locally finite collection $V$ of open sets which is a refinement of $K_{p}$ and covers $L$. The collection $U+V$ is a locally finite collection of open sets which is a refinement of $K_{p}$ and covers $M_{p}$. It follows that $X$ is countably paracompact.

\section{REFERENCES}

1. G. Birkhoff, Lattice theory, Amer. Math. Soc. Colloquium Publications, vol. 25, rev. ed., New York, 1948.

2. J. Dieudonné, Une generalization des espaces compacts, J. Math. Pures Appl. vol. 23 (1944) pp. 65-76.

3. C. H. Dowker, On countably paracompact spaces, Canadian Journal of Mathematics vol. 3 (1951) pp. 175-186.

UNIVERSITY OF VIRGINIA 